\title{
La importancia del financiamiento sobre la producción científica en México
}

\author{
Eva Ugarte Pineda* \\ Gilberto Parra Huerta*
}

\begin{abstract}
Artículo recibido:
9 de agosto de 2020

Artículo aceptado:

12 de noviembre de 2020

Artículo de investigación
\end{abstract}

\section{Resumen}

En esta investigación se estudia el financiamiento de la investigación básica en México en el periodo 2009. 2019. El análisis utiliza datos de Web of Science y se enfoca en el Science Citation Index Expanded. Ponemos especial atención en el impacto que tienen los recursos del Conacyt sobre la producción académica. Los resultados revelan que $75.65 \%$ de las publicaciones del periodo recibieron financiación. El Conacyt aparece como institución financiadora en $46.05 \%$ de los documentos. Otro aspecto que estudiamos es la difusión de la investigación científica. Identificamos que $68.13 \%$ de los documentos que recibieron apoyo económico del Conacyt se publicaron en revistas de acceso restringido, es decir, están detrás de muros de pago.

\footnotetext{
Departamento de Economía, Universidad Autónoma Metropolitana, Unidad Azcapotzalco, México eup@azc.uam.mx gph@azc.uam.mx 
Palabras clave: Financiamiento de la Ciencia; Investigación Básica; Conacyt

The relevance of funding on scientific production in Mexico

Eva Ugarte Pineda and Gilberto Parra Huerta

\begin{abstract}
The aim of this paper is to explore the funding of basic research in Mexico during the period 2009-2019. The study uses data from the Web of Science and focuses on the Science Citation Index Expanded. We pay special attention to the impact that Conacyt's economic support has on academic production. Our analysis reveals that $75.65 \%$ of the publications received funding. Conacyt appears as funding institution in $46.05 \%$ of these documents. We also investigated the dissemination of scientific research. We found that $68.13 \%$ of the documents that received financial support from Conacyt were published in restricted access journals, that is, that they are behind paywalls.
\end{abstract}

Keywords: Funding Science; Basic Research; Conacyt

\title{
INTRODUCCIÓN
}

L a Web of Science ha sido utilizada en investigaciones previas para estudiar el financiamiento a la producción científica en diversos países. Sus resultados muestran que las naciones desarrolladas y emergentes financian una parte considerable de sus investigaciones en ciencia básica (Huang y Huang, 2018; Wang et al., 2012; Xu, Tan y Zhao, 2015). Por ejemplo, en el periodo 2009-2010 China subvencionó 76.1 \% de sus artículos, Estados Unidos $64.2 \%$, Brasil $64 \%$, Alemania $57.2 \%$, España $68.3 \%$, India $45.2 \%$ y Francia 51.7 \% (Tan, Zhao y Ye, 2012). En lo que respecta a México, en la literatura académica se cuenta con poca información sobre la tendencia y la estructura del financiamiento de la actividad científica. Este artículo pretende abonar a cubrir ese vacío de información.

El objetivo central de la investigación estriba en responder tres preguntas concretas: ¿qué porcentaje de las publicaciones mexicanas recibieron 
recursos financieros, públicos o privados? ¿Qué porcentaje de los artículos nacionales recibieron recursos del Conacyt? ¿Qué porcentaje de los artículos mexicanos, financiados por el Conacyt, se publicaron en revistas de acceso abierto? Esta última pregunta está relacionada con el acceso abierto, que se ha convertido en un tema de interés para los académicos y agencias de financiamiento. Un objetivo adicional de la investigación es mostrar que los datos de la Web of Science representan una ventana de oportunidad para estudiar la estructura y la tendencia del financiamiento de la ciencia en México.

El trabajo tiene la siguiente estructura: en la sección segunda describimos la estrategia de búsqueda que utilizamos para identificar las publicaciones nacionales en la Web of Science; en la tercera sección sintetizamos los hallazgos en varios gráficos y tablas, para discutir diversos temas en la cuarta sección: primero examinamos el vínculo entre el financiamiento y la producción académica, después exponemos la forma en que la teoría económica justifica la participación del Estado en el desarrollo científico y, por último, explicamos la importancia del acceso abierto. En las conclusiones sintetizamos los principales resultados del trabajo y señalamos que los datos de la Web of Science pueden mejorar nuestro entendimiento sobre la dinámica del financiamiento en nuestro país.

\section{Metodología}

En 2008 la Web of Science comenzó a ofrecer información sobre el financiamiento recibido por las publicaciones indizadas en su base de datos. Varios académicos vieron en esta información una ventana de oportunidad para estudiar y entender las tendencias y la estructura de la financiación a la investigación científica. Por ejemplo, Zhao et al. (2016) examinaron los patrones de financiación en el campo económico; Serôdio, McKee y Stuckler (2018) se enfocaron en los recursos provistos por Coca-Cola a la investigación; Xu, Tan y Zhao (2015) estudiaron las subvenciones en el campo de las ciencias sociales, y Álvarez-Bornstein, Díaz-Faes y Bordons (2019) estudiaron los patrones de financiamiento en la investigación biomédica.

La Web of Science registra información sobre el financiamiento para dos de sus índices: el Science Citation Index Expanded (SCIE), que comenzó a indizar la información en 2008, y el Social Science Citation Index, que inició en 2015. Solamente se registra información para dos tipos de documentos: artículos y revisiones (Álvarez-Bornstein, Morillo y Bordons, 2017; PaulHus, Desrochers y Costas, 2016). En ambos índices dominan las publicaciones en idioma inglés; según cálculos de Liu, Tang y Hu (2020) 99.70 \% de los 
artículos indizados en el SCIE que reportan haber recibido financiamiento se escribieron en dicho idioma.

Los datos sobre el financiamiento que ofrece la Web of Science están construidos con base en la información que reportan los autores. Por ejemplo, Ostrovsky, Rickenstorff-Parrao y Arrizón (2013: 536) mencionan lo siguiente en la sección de agradecimientos: "This work was supported by the Benemérita Universidad Autónoma de Puebla (project VIEP OSA-EXC-13) and by the National Council for Science and Technology of Mexico (project CB-165142)".

La Web of Science extrae del texto de financiamiento dos datos: el nombre de la entidad que otorgó la subvención y el número de concesión. A partir de esos datos construye una tabla similar a la Tabla 1.

\begin{tabular}{|c|c|}
\hline Entidad financiadora & Número de concesión \\
\hline Benemérita Universidad Autónoma de Puebla & VIEP OSA-EXC-13 \\
\hline $\begin{array}{c}\text { Consejo Nacional de Ciencia y Tecnología } \\
\text { (CONACyT) }\end{array}$ \\
$\begin{array}{c}\text { Aparece en contenido como } \\
\text { National Council for Science and Technology of } \\
\text { México }\end{array}$ & CB-165142 \\
\hline
\end{tabular}

*Nombre preferido asignado por la Web of Science

Tabla 1. Datos sobre el financiamiento reportados por Antonio-Lopez et al. (2014)

Las entidades financiadoras pueden aparecer bajo diferentes denominaciones. Por ejemplo, en el artículo de Antonio-Lopez et al. (2014: 4311) el Conacyt aparece como "Mexican Council of Science and Technology (CONACyT)". La Web of Science agrupa las diversas denominaciones que hacen referencia a una misma entidad financiadora bajo un nombre preferido. Sin embargo, algunas publicaciones que reportan haber recibido financiamiento del Conacyt no son agrupadas bajo ese nombre preferido. Por ejemplo, el artículo de Ramirez-Solis et al. (2018: 2849) señala siguiente en los agradecimientos: "A.R.S. thanks support from CONACYT Basic Science Project number 253679. A.EJ.I. thanks a DGAPA-UNAM postdoctoral fellowship. J.H.C. thanks support from DGAPA-UNAM grant No. IG100416”.

En este caso la Web of Science identifica la entidad financiadora como “CONACYT Basic Science Project" (Tabla 2). Por tanto, si buscamos los artículos subvencionados por el Conacyt utilizando únicamente el nombre preferido, el artículo de Ramirez-Solis et al. (2018) y otros podrían quedar fuera 
de nuestro conjunto de datos. En ese contexto, para minimizar la pérdida de información se decidió trabajar con todos los documentos que contuvieran el acrónimo Conacyt en el campo denominado entidad financiadora.

\begin{tabular}{|c|c|}
\hline Entidad financiadora & Número de concesión \\
\hline CONACYT Basic Science Project & 253679 \\
\hline Universidad Nacional Autónoma de México & IG100416 \\
\hline
\end{tabular}

Tabla 2. Datos sobre el financiamiento reportados por Ramirez-Solis et al. (2018)

En esta investigación se trabajó con datos del SCIE para tener un horizonte temporal más amplio. La estrategia de búsqueda se limitó solamente a los artículos y revisiones publicados en idioma inglés. La búsqueda abarcó varias etapas e incluyó el uso de algunas etiquetas de campo. La estrategia que seguimos para identificar las publicaciones se basó en los trabajos de Huang y Huang (2018) y Wang et al. (2012).

\begin{tabular}{|c|c|c|}
\hline & Campo de búsqueda & Artículos \\
\hline$\# 1$ & CU=México & 128689 \\
\hline \#2 & 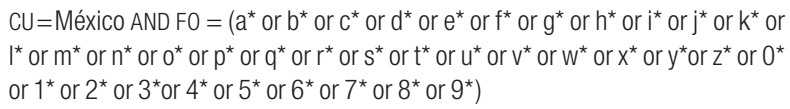 & 97357 \\
\hline \#3 & $C U=$ México AND F0 = Conacyt & 59267 \\
\hline \#4 & (\#2 NOT \#3) & 38090 \\
\hline \#5 & (\#1 NOT \#2) & 31332 \\
\hline
\end{tabular}

Tabla 3. Estrategia de búsqueda

En la Tabla 3 se expone la estrategia seguida para buscar las publicaciones. ${ }^{1}$ A través de la búsqueda \#1 identificamos todos los documentos indizados en el SCIE en los que participan investigadores de México. La \#2 nos permitió localizar todos los documentos que recibieron financiamiento (público o privado) para su realización. La búsqueda \#3 abarca todos los documentos que recibieron fondos del Conacyt. La \#4 contempla todas las publicaciones que recibieron fondos de una entidad diferente al Conacyt.

1 La etiqueta de campo CU permite identificar las publicaciones pertenecientes a un país específico. La etiqueta FO permite identificar a las agencias financiadoras. 
La \#5 abarca los documentos que no recibieron recursos económicos para su realización. En total identificamos 128689 publicaciones nacionales en el SCIE.

La información obtenida se descargó a través de una herramienta que ofrece la Web of Science denominada análisis de los resultados. Las variables que utilizamos fueron las siguientes: 1) años de publicación, 2) organización-consolidada, y 3) categorías de Web of Science. La variable organización-consolidada brinda información sobre la producción científica a nivel institucional. Los autores suelen reportar su adscripción institucional de forma distinta, por ende, la base de datos agrupa las diversas denominaciones de una institución bajo un nombre único que designa organización-consolidada. Por otra parte, las categorías ofrecen información sobre las áreas científicas donde aparecen las publicaciones buscadas. La Web of Science clasifica cada publicación en una o más áreas científicas. Esta información nos brinda una visión esquemática de los campos del conocimiento donde se enfoca la investigación nacional.

\section{Limitaciones}

Es importante señalar que la información que ofrece la Web of Science no es $100 \%$ precisa. Álvarez-Bornstein, Morillo y Bordons (2017) examinaron la precisión de los datos de la Web of Science en 1045 publicaciones españolas. Los autores detectaron una pérdida de información en $12 \%$ de los documentos. Asimismo, señalan que la información sobre las entidades y el número de concesión no siempre es consistente.

Asimismo, la Web of Science tiene limitaciones en su cobertura que pueden dar lugar a lecturas sesgadas del estado de la producción científica y el grado de financiamiento de una institución o país. En la literatura bibliométrica se pueden consultar varios estudios que dan cuenta de la poca representatividad que tienen las revistas latinoamericanas en dicha base de datos (Aguado-López et al., 2014; Mongeon y Paul-Hus, 2016; Santa y Herrero-Solana, 2010). Esto implica que si los investigadores publican en revistas no indexadas los artículos no se contabilizarán. En ese sentido, es importante tomar los resultados con una perspectiva crítica, sobre todo si serán utilizados en la formulación de políticas de ciencia y tecnología. 


\section{Presentación y ANÁlisis DE RESUltados}

En la Figura 1 vemos que la producción académica registrada en el SCIE ha crecido en forma exponencial en el periodo que abarca de 2009 a 2019. Esta tendencia se explica en gran medida por el financiamiento que han recibido los investigadores. En el periodo se publicaron 128689 artículos y $75.65 \%$ de estos recibieron recursos económicos para su desarrollo. En dicha figura comparamos dos curvas: la tendencia de los artículos con financiamiento y sin financiamiento. Se puede observar que las curvas presentan una diferencia notable en la velocidad de su crecimiento. Las investigaciones con recursos crecieron a una tasa anual promedio de $10.08 \%$; por su parte, aquellas que no recibieron apoyo financiero crecieron a una tasa de $2.64 \%$. Inclusive se observa que la producción sin financiamiento decrece en algunos años. En síntesis, la figura revela que en ausencia de financiación la investigación nacional hubiera permanecido prácticamente estancada.

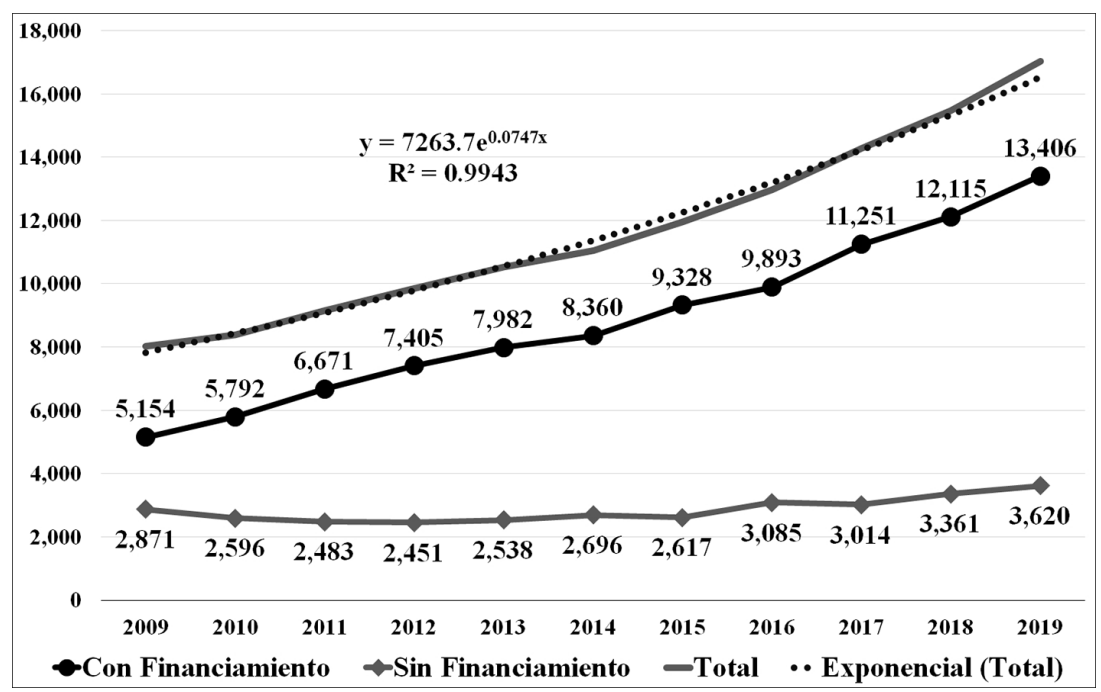

Figura 1. Tendencia de los artículos con financiamiento, público o privado, y sin financiamiento en el periodo 2009-2019

Fuente: elaboración propia con datos de la Web of Science

En la Figura 2 examinamos el papel del Conacyt sobre la producción académica nacional. Observamos que en el periodo 2009-2019 46.05 \% de los documentos publicados por investigadores mexicanos recibieron recursos de dicho organismo. La relevancia del Conacyt ha crecido año con año. En 2009 $37.53 \%$ de las publicaciones recibieron fondos del instituto, mientras que en 
2019 los recibieron 47.06 \%; un aumento de 9.52 puntos porcentuales. En la misma figura también se observa que otras agencias de financiamiento han ganado terreno en el periodo. En 2009 otras agencias financiaron $26.69 \%$ de las investigaciones, mientras que en 2019 subvencionaron $31.68 \%$.

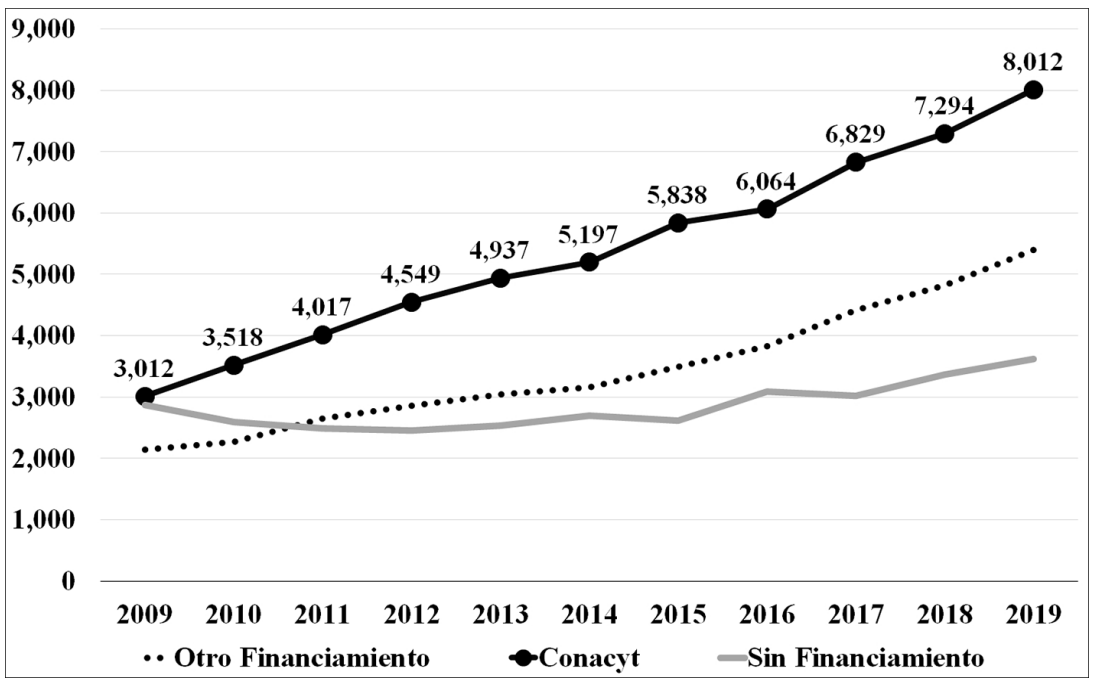

Figura 2. Tendencia de los artículos que recibieron fondes del Conacyt Fuente: elaboración propia con datos de la Web of Science

La Tabla 4 presenta información sobre las 15 instituciones nacionales que más artículos tienen registrados en el SCIE. Observamos que el financiamiento público y privado tiene un fuerte impacto sobre la producción científica de las universidades. La Tabla 5 muestra la estructura del financiamiento en las áreas científicas donde más publican los investigadores nacionales. Se observa que algunos campos, como la Astronomía y Astrofísica, son muy dependientes de las subvenciones.

En la Figura 3 podemos observar bajo cuál esquema se publica la información científica generada en México. El 31.78 \% de los artículos que recibieron recursos del Conacyt se encuentran en acceso abierto. El $41.62 \%$ de los documentos que obtuvieron recursos de otras fuentes de financiamiento se encuentran disponibles de forma gratuita. Por último, $26.39 \%$ de las publicaciones que no reportan financiamiento de ningún tipo se encuentran en acceso abierto. Esta figura muestra que la mayor parte de las investigaciones que utilizaron fondos públicos se encuentran detrás de muros de pago. 


\begin{tabular}{|l|c|c|c|c|}
\hline \multicolumn{1}{|c|}{ Institución } & SF\% & OF\% & Conacyt $\%$ & Total \\
\hline $\begin{array}{l}\text { Universidad Nacional Autónoma de México } \\
\text { (UNAM) }\end{array}$ & 16.73 & 31.51 & 51.76 & 40,270 \\
\hline Instituto Politécnico Nacional & 19.39 & 20.76 & 59.85 & 15,795 \\
\hline $\begin{array}{l}\text { Centro de Investigación y de Estudios Avanza- } \\
\text { dos (CINVESTAV) }\end{array}$ & 16.63 & 13.84 & 69.53 & 11,035 \\
\hline Universidad Autónoma Metropolitana & 28.20 & 19.90 & 51.90 & 7,212 \\
\hline $\begin{array}{l}\text { Benemérita Universidad Autónoma de Puebla } \\
\text { (BUAP) }\end{array}$ & 17.47 & 20.54 & 62.00 & 5,010 \\
\hline Universidad Autónoma de Nuevo León & 30.93 & 26.61 & 42.46 & 4,656 \\
\hline Universidad de Guadalajara & 29.20 & 26.48 & 44.32 & 4,486 \\
\hline $\begin{array}{l}\text { Universidad Autónoma de San Luis Potosí } \\
\text { (UASLP) }\end{array}$ & 17.71 & 17.98 & 64.30 & 4,460 \\
\hline Tecnológico de Monterrey & 27.91 & 32.83 & 39.26 & 3,698 \\
\hline Instituto Mexicano del Seguro Social & 33.66 & 29.48 & 36.86 & 3,277 \\
\hline Universidad de Guanajuato & 18.82 & 24.47 & 56.71 & 3,236 \\
\hline Universidad Autónoma del Estado de Morelos & 19.91 & 20.01 & 60.08 & 3,184 \\
\hline $\begin{array}{l}\text { Universidad Michoacana de San Nicolás } \\
\text { de Hidalgo (UMSNH) }\end{array}$ & 17.81 & 20.64 & 61.55 & 2,926 \\
\hline Universidad Veracruzana & 24.10 & 25.08 & 50.82 & 2,548 \\
\hline Universidad Autónoma de Baja California & 26.63 & 27.39 & 45.99 & 2,505 \\
\hline
\end{tabular}

$\mathrm{SF}=$ Sin Financiamiento; $\mathrm{OF}=0$ tro Financiamiento (público o privado)

Tabla 4. Estructura del financiamiento de las principales instituciones mexicanas Fuente: elaboración propia con datos de la Web of Science

\begin{tabular}{|l|c|c|c|c|}
\hline \multicolumn{1}{|c|}{ Categoría } & SF\% & OF\% & Conacyt $\%$ & Total \\
\hline Ciencias de los Materiales, Multidisciplinaria & 20.54 & 23.05 & 56.41 & 6,976 \\
\hline Ciencias Ambientales & 20.65 & 35.82 & 43.53 & 5,686 \\
\hline Química Física & 16.09 & 23.21 & 60.70 & 5,532 \\
\hline Astronomía y Astrofísica & 7.08 & 38.27 & 54.65 & 5,477 \\
\hline Bioquímica y Biología Molecular & 12.06 & 26.19 & 61.74 & 5,322 \\
\hline Ciencias Vegetales & 17.86 & 35.84 & 46.30 & 4,721 \\
\hline Ingeniería, Eléctrica y Electrónica & 34.07 & 19.09 & 46.84 & 4,464 \\
\hline Física Aplicada & 20.62 & 22.21 & 57.17 & 4,259 \\
\hline Química, Multidisciplinaria & 14.20 & 24.12 & 61.68 & 4,084 \\
\hline Ciencia y Tecnología de los Alimentos & 23.81 & 24.31 & 51.89 & 4,003 \\
\hline
\end{tabular}




\begin{tabular}{|l|c|c|c|c|}
\hline Ingeniería Química & 26.63 & 20.31 & 53.06 & 3,890 \\
\hline Ciencias Multidisciplinarias & 11.01 & 42.06 & 46.93 & 3,652 \\
\hline Biotecnología y Microbiología Aplicada & 15.09 & 26.91 & 58.00 & 3,552 \\
\hline Ecología & 12.38 & 42.43 & 45.20 & 3,434 \\
\hline Física, Multidisciplinaria & 24.30 & 23.73 & 51.97 & 3,346 \\
\hline
\end{tabular}

$\mathrm{SF}=\mathrm{Sin}$ Financiamiento; OF=0tro Financiamiento (público o privado)

Tabla 5. Estructura del financiamiento de las principales áreas científicas

Fuente: elaboración propia con datos de la Web of Science

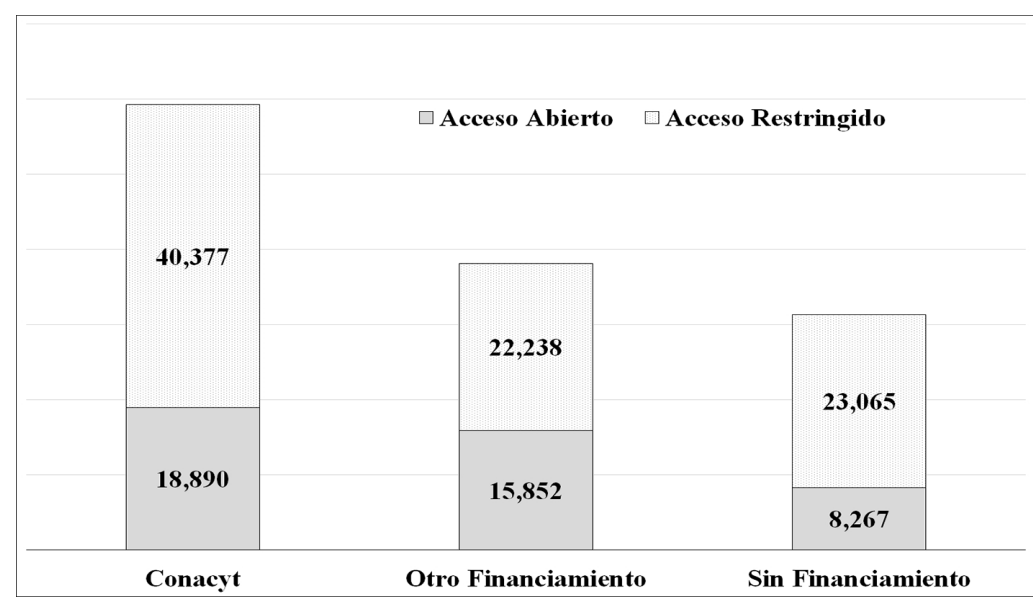

Figura 3. Publicaciones que se encuentran en acceso abierto y acceso restringido por tipo de financiamiento recibido en el periodo 2009-2019 Fuente: elaboración propia con datos de la Web of Science

\section{Discusión}

¿Las subvenciones inciden sobre la productividad de los académicos? Varias investigaciones previas han mostrado que existe una relación positiva entre el financiamiento y la producción científica. Por ejemplo, Payne y Siow (2003) examinaron el efecto que tienen los recursos federales sobre la producción científica de 68 universidades de los Estados Unidos. Los autores afirman que un millón de dólares en fondos se traduce en 10 artículos más. Rosenbloom et al. (2015) midieron el impacto de la financiación sobre la productividad científica de los académicos del área de Química en los Estados Unidos. Sus hallazgos muestran que un millón de dólares en financiamiento se traduce en seis o siete artículos más. Benavente et al. (2012) usaron una 
regresión discontinua para medir el efecto que tienen los recursos del Fondo Nacional de Desarrollo Científico y Tecnológico sobre la producción de los académicos en Chile. Los autores encontraron que después de la recepción de la subvención se producen dos publicaciones adicionales en el plazo de seis años.

En lo que respecta a México, los datos nos señalan que existe una fuerte asociación entre las subvenciones y la producción científica. En el periodo 2009-2019, $76.65 \%$ de los artículos generados en el país recibieron recursos económicos, esto significa que en ausencia de financiación la producción académica hubiera permanecido prácticamente estancada. En otros países se han identificado cifras similares; Huang y Huang (2018) muestran que en el periodo 2009-2014 China subvencionó 81.55 \% de sus artículos, Estados Unidos 60.44 \% , Alemania $59.65 \%$, España $68.3 \%$ y Francia 51.7 \%.

\section{Financiamiento público de la ciencia y la teoría económica}

Los datos de México y otros países muestran que los organismos públicos son generalmente quienes más recursos proveen a la ciencia básica. ¿Por qué razón el Estado tiene este rol protagónico? Desde la teoría económica se ofrece una respuesta a esta interrogante (Arrow, 1962; Nelson, 1959).

El conocimiento es un bien público que puede definirse a partir de dos atributos: la no rivalidad y la no exclusión (Hess y Ostrom, 2003; Stiglitz, 1999). Foray (2004) divide la no rivalidad en dos niveles: individual y colectiva. La no rivalidad en el plano individual implica que un agente puede utilizar una pieza de conocimiento un sinnúmero de veces sin incurrir en un gasto. Por ejemplo, una persona puede pagar por un curso para aprender una técnica de cultivo y posteriormente puede aplicar de forma reiterada esa técnica sin incurrir en ningún costo adicional. En el plano colectivo la no rivalidad implica que varios agentes pueden usar el conocimiento de forma simultánea. Por ejemplo, dos o más médicos pueden utilizar de forma simultánea un mismo procedimiento quirúrgico.

La no exclusión significa que no podemos limitar el uso que otros agentes hacen del conocimiento. Por ejemplo, los métodos matemáticos no pueden patentarse en México, por lo tanto, si un matemático descubre un nuevo método y lo publica en una revista científica las empresas pueden usarlo para desarrollar innovaciones sin incurrir en ningún costo. No obstante, existen conocimientos que sí pueden patentarse y los propietarios pueden ejercer el derecho de exclusión; por ejemplo, una batería recargable que combine de forma novedosa varios elementos químicos. Por lo anterior, se considera que el conocimiento es un bien público impuro. 
La teoría económica afirma que los bienes públicos son una de las causas detrás de los fallos de mercado. Arrow (1962) menciona tres factores que conducen a fallas de mercado: la indivisibilidad, la no apropiabilidad y la incertidumbre. En el caso del conocimiento se presentan los tres factores. $\mathrm{La}$ indivisibilidad implica que el conocimiento tiene que pagarse de forma íntegra independientemente de la cantidad que va a utilizarse (Warsh, 2006). La indivisibilidad es una característica que complica el intercambio del conocimiento en el mercado, pues es difícil para el demandante estimar la utilidad de una pieza de conocimiento sin antes conocerla por completo. La no apropiabilidad implica que las firmas tienen dificultades para capturar a través del mercado los beneficios generados por sus conocimientos producidos. Por último, la incertidumbre implica que la producción de conocimiento es un proceso altamente impredecible (Arrow, 1962). "Debido a la incertidumbre la investigación no puede ser gestionada y evaluada de la misma forma que la producción de bienes y servicios. Esto implica que la investigación depende menos de la rentabilidad y la entrega oportuna que otras actividades" (Foray, 2004: 53).

La existencia de fallas de mercado implica que las fuerzas del mercado no pueden generar una cantidad socialmente óptima de conocimiento. Las firmas perciben la investigación como un proceso altamente riesgoso y además enfrentan dificultades para capturar los beneficios de sus descubrimientos, por lo tanto, invierten una cantidad insuficiente de recursos en la producción de nuevos conocimientos. En este contexto, la "asignación óptima a la invención requiere que el gobierno o algún otro organismo no regido por el criterio de ganancias y pérdidas financie la investigación y la invención" (Arrow, 1962: 623).

Wang et al. (2012), utilizando datos del SCIE, calcularon para 2009 la proporción de artículos financiados por entes públicos en 10 países. Sus resultados muestran que en Estados Unidos los Institutos Nacionales de Salud Pública financiaron $19.87 \%$ de sus publicaciones y la Fundación Nacional de Ciencia 11.84 \%; en China la Fundación de Ciencias Naturales financió $63 \%$ de los artículos publicados mientras que en España el Ministerio de Educación y Ciencia proveyó de fondos a $17.16 \%$ de las investigaciones y el Ministerio de Ciencia e Innovación a 7.66 \% . De acuerdo con nuestros datos, en México el Conacyt financió $37.53 \%$ de los documentos que se publicaron en 2009. Estas cifras ponen de relieve que los entes públicos son un actor central en la promoción de la ciencia básica. 


\section{La relevancia del acceso abierto}

El stock de conocimiento de una economía crece de forma eficiente cuando la información circula sin restricciones, ya que ésta no es solamente un producto sino también un insumo necesario para el desarrollo de nuevas investigaciones (Hess y Ostrom, 2003). Para alcanzar "una eficiencia económica estática máxima, el conocimiento debe administrarse como un bien común, con acceso libre para todos los que puedan usarlo" (Nelson, 1959: 306). Sin embargo, en los últimos años gran parte del conocimiento generado se ha convertido en un bien privado.

Un artículo se considera de acceso abierto cuando se puede consultar, copiar, imprimir y distribuir libremente sin costo (Abadal, 2012). En 2013 solamente $11 \%$ de las publicaciones indizadas en la Web of Science eran de acceso abierto (Torres-Salinas y Orduña-Malea, 2014). La privatización del conocimiento puede limitar fuertemente el avance social, ambiental y económico de los países en desarrollo. Además, acceder a la literatura científica de vanguardia requiere de una amplia capacidad económica y eso genera una gran asimetría entre las naciones en lo que respecta al acceso al conocimiento (Kirsop y Chan, 2005). Algunos países están tomando medidas para evitar la privatización del conocimiento. En 2018, 11 organizaciones miembros de la Science Europe pusieron en marcha el denominado Plan S, el cual pretende que todas las investigaciones financiadas por la Science Europe publiquen sus resultados bajo un esquema de acceso abierto (Else, 2018). No obstante, también existen críticas a dicho plan.

El Plan S mantiene intacto el monopolio de las grandes editoriales. Asimismo, el modelo basado en "cargos por procesamiento de artículos" puede alterar el modelo editorial que prima en América Latina (libre de costos para el autor y lector), pues las revistas locales pueden optar por un sistema similar basado en el cobro a los autores (Debat y Babini, 2020). Por otra parte, el costo por publicar en acceso abierto puede ser prohibitivo para la mayoría de los investigadores de América Latina (Noorden, 2020), región que tiene problemas recurrentes para invertir de forma suficiente en ciencia y tecnología.

En el caso de México, $68.13 \%$ de los artículos que recibieron fondos del Conacyt se publicaron en revistas de acceso restringido. Esto significa que gran parte de la población mexicana no tiene acceso a los resultados de las investigaciones financiadas con recursos del Estado. Los repositorios institucionales impulsados por el Conacyt representan un esfuerzo loable en aras de la difusión de la información académica; sin embargo, también es importante discutir la posibilidad de poner a disposición de los mexicanos de forma gratuita e inmediata las investigaciones que reciben subvenciones del Conacyt. 


\section{Conclusiones}

Los resultados nos permiten alcanzar varias conclusiones importantes: 1) El financiamiento público y privado tiene un fuerte impacto sobre la producción científica nacional. 2) El Conacyt tiene un rol relevante sobre la producción académica en México, pues el instituto financió $46.05 \%$ de las publicaciones en el periodo 2009-2019. 3) El 68.13 \% de las publicaciones que recibieron recursos del Conacyt son inaccesibles para la mayoría de los mexicanos pues se encuentran detrás de muros de pago. De acuerdo con la teoría económica esta circulación restringida del conocimiento puede minar el avance de la ciencia básica y la innovación.

Un objetivo del artículo era mostrar que los datos de la Web of Science representan una ventana de oportunidad para estudiar diferentes aspectos de la estructura y la tendencia de financiación en el país. La base de datos nos permite también abordar otros temas; por ejemplo, la forma en que se distribuyen los fondos entre hombres y mujeres, la relación entre los fondos públicos y el número de citas que reciben los artículos, el vínculo entre el financiamiento y la colaboración internacional o la forma en que se reparten los recursos entre las universidades. Indagar en estos tópicos, sin duda, contribuirá a que los encargados de la política científica tomen mejores decisiones en el diseño y asignación de los fondos a la investigación básica.

\section{REFERENCIAS}

Abadal, Ernest. 2012. Acceso Abierto a la Ciencia. Barcelona: UOC.

Aguado-Lopez, Eduardo, Arianna Becerril-García, Miguel Arriola y Néstor Martínez-Domínguez. 2014. "Iberoamérica en la Ciencia de Corriente Principal (Thomson Reuters /Scopus): una region fragmentada". Interciencia 39 (8): 570-579.

Álvarez-Bornstein, Belén, Adrián Díaz-Faes y María Bordons. 2019. "What Characterises Funded Biomedical Research? Evidence from a Basic and a Clinical Domain". Scientometrics 119 (2): 805-825. https://doi.org/10.1007/s11192-019-03066-3

Álvarez-Bornstein, Belén, Fernanda Morillo y María Bordons. 2017. "Funding Acknowledgments in the Web of Science: Completeness and Accuracy of Collected Data". Scientometrics 112 (3): 1793-1812. https://doi.org/10.1007/s11192-017-2453-4

Antonio-Lopez, J. Enrique, Zeinab Sanjabi Eznaveh, Patrick LiKamWa, Axel Schülzgen y Rodrigo Amezcua-Correa. 2014. "Multicore Fiber Sensor for High-Temperature Applications up to $1000^{\circ} \mathrm{C}$ ”. Optics Letters 39 (15): 4309-4312. https://doi.org/10.1364/OL.39.004309 
Arrow, Kenneth. 1962. "Economic Welfare and the Allocation of Resources for Invention". En National Bureau of Economical Research: The Rate and Direction of Inventive Activity: Economic and Social Factors, 609-626. http://www.nber.org/chapters/c2144.pdf

Benavente, José, Gustavo Crespi, Lucas Figal Garone y Alessandro Maffioli. 2012. "The Impact of National Research Funds: A Regression Discontinuity Approach to the Chilean FONDECYT”. Research Policy 41 (8): 1461-1475. https://doi.org/10.1016/j.respol.2012.04.007

Debat, Humberto y Dominique Babini. 2020. "Plan S in Latin America: A Precautionary Note". Scholarly and Research Communication 11 (1): 1-12. https://doi.org/10.22230/src.2020v11n1a347

Else, Holly. 2018. "Radical Plan to End Paywalls". Nature 561: 17-18. https://doi.org/10.1038/d41586-018-06178-7

Foray, Dominique. 2004. The Economics of Knowledge. Massachusetts: MIT Press.

Hess, Charlotte y Elinor Ostrom. 2003. "Ideas, Artifacts, and Facilities: Information as a Common-Pool Resource”. Law and Contemporary Problems 66 (1/2): 111-145. http://www.jstor.org/stable/20059174

Huang, Mu-Hsuan y Mei-Jhen Huang. 2018. "An Analysis of Global Research Funding from Subject Field and Funding Agencies Perspectives in the G9 Countries". Scientometrics 115 (2): 833-847. https://doi.org/10.1007/s11192-018-2677-y

Kirsop, Barbara y Leslie Chan. 2005. "Transforming Access to Research Literature for Developing Countries”. Serials Review 31 (4): 246-255. https://doi.org/10.1016/j.serrev.2005.09.003

Liu, Weishu, Li Tang y Guangyuan Hu. 2020. "Funding Information in Web of Science: An Updated Overview”. Scientometrics 122: 1509-1524. https://doi.org/10.1007/s11192-020-03362-3

Mongeon, Philippe y Adèle Paul-Hus. 2016. "The Journal Coverage of Web of Science and Scopus: A Comparative Analysis”. Scientometrics 106 (1): 213-228. https://doi.org/10.1007/s11192-015-1765-5

Nelson, Richard R. 1959. “The Simple Economics of Basic Scientific Research”. Journal of Political Economy 67 (3): 297-306. https://doi.org/10.1086/258177

Noorden, Richard Van. 2020. "Nature Journals Announce First Open-Access Agreement”. Nature (October). https://doi.org/10.1038/d41586-020-02959-1

Ostrovsky, Andrey S., Carolina Rickenstorff-Parrao y Víctor Arrizón. 2013. “Generation of the 'Perfect' Optical Vortex Using a Liquid-Crystal Spatial Light Modulator”. Optics Letters 38 (4): 534-536. https://doi.org/10.1364/OL.38.000534

Paul-Hus, Adèle, Nadine Desrochers y Rodrigo Costas. 2016. "Characterization, Description, and Considerations for the Use of Funding Acknowledgement Data in Web of Science". Scientometrics 108 (1): 167-182. https://doi.org/10.1007/s11192-016-1953-y

Payne, A. Abigail y Aloysius Siow. 2003. "Does Federal Research Funding Increase University Research Output?”. Advances in Economic Analysis E Policy 3 (1). https://doi.org/10.2202/1538-0637.1018 
Ramirez-Solis, Alejandro, Jorge Iván Amaro-Estrada, Jorge Hernández-Cobos y Laurent Maron. 2018. "Aqueous Solvation of SmI 3: A Born-Oppenheimer Molecular Dynamics Density Functional Theory Cluster Approach”. Inorganic Chemistry 57 (5): 2843-2850.

https://doi.org/10.1021/acs.inorgchem.7b03220

Rosenbloom, Joshua L., Donna K. Ginther, Ted Juhl y Joseph A. Heppert. 2015. "The Effects of Research \& Development Funding on Scientific Productivity: Academic Chemistry, 1990-2009”. PLOS ONE 10 (9): e0138176. https://doi.org/10.1371/journal.pone.0138176

Santa, Samaly y Víctor Herrero-Solana. 2010. "Cobertura de la Ciencia de América Latina y El Caribe en Scopus vs Web of Science”. Investigacion Bibliotecologica 24 (52): 13-27. https://doi.org/10.22201/iibi.0187358xp.2010.52.27451

Serôdio, Paulo M, Martin McKee y David Stuckler. 2018. "Coca-Colan- a Model of Transparency in Research Partnerships? A Network Analysis of Coca-Cola's Research Funding (2008-2016)". Public Health Nutrition 21 (9): 1594-1607. https://doi.org/10.1017/S136898001700307X

Stiglitz, Joseph E. 1999. "Knowledge as a Global Public Good”, en Global Public Goods, editado por Inge Kaul, Isabelle Grunberg y Marc Stern, 308-325. New York: Oxford University Press. https://doi.org/10.1093/0195130529.003.0015

Tan, Alice M., Star X. Zhao y Fred Y. Ye. 2012. "Funds Promote Scientific Output". Current Science 102 (4): 542-543. https://www.currentscience.ac.in/cs/Volumes/102/04/0542.pdf

Torres-Salinas, Daniel y Enrique Orduña-Malea. 2014. "Ruta Dorada del Open Access en Web of Science”. Anuario ThinkEPI 8: 211-214. https://recyt.fecyt.es/index.php/ThinkEPI/article/view/29580

Wang, Xianwen, Di Liu, Kun Ding y Xinran Wang. 2012. "Science Funding and Research Output: A Study on 10 Countries". Scientometrics 91 (2): 591-599. https://doi.org/10.1007/s11192-011-0576-6

Warsh, David. 2006. El conocimiento y la riqueza de las naciones: el enigma del crecimiento económico, su historia y su explicación moderna. España: Antoni Bosch.

Xu, Xin, Alice Tan y Star Zhao. 2015. "Funding Ratios in Social Science: The Perspective of Countries/Territories Level and Comparison with Natural Sciences". Scientometrics 104 (3): 673-684. https://doi.org/10.1007/s11192-015-1633-3

Zhao, Star X., Shuang Yu, Alice M. Tan, Xin Xu y Haiyan Yu. 2016. “Global Pattern of Science Funding in Economics”. Scientometrics 109 (1): 463-479. https://doi.org/10.1007/s11192-016-1961-y

Para citar este texto:

Ugarte Pineda, Eva y Gilberto Parra Huerta. 2021. "La importancia del financiamiento sobre la producción científica en México”. Investigación Bibliotecológica: archivonomía, bibliotecología e información 35 (87): 187-202. http://dx.doi.org/10.22201/iibi.24488321xe.2021.87.58330 